\title{
Highly efficient SERS inside microstructured optical fibres via optical mode engineering
}

\author{
A. C. Peacock, A. Amezcua-Correa, P. J. A. Sazio \\ Optoelectronics Research Centre, University of Southampton, SO17 1BJ, UK \\ J. J. Baumberg \\ School of Physics and Astronomy, University of Southampton, SO17 1BJ, UK
}

J. Yang and S. M. Howdle

School of Chemistry, University of Nottingham, NG7 2RD, UK

\begin{abstract}
Microstructured optical fibres (MOFs) offer versatile engineering of the internal microstructure geometry to provide large surface areas and aspect ratios with outstanding mechanical properties which, when functionalised with metal nanoparticles, serve as exceptional substrates for surface enhanced Raman spectroscopy (SERS) due to the large electromagnetic fields generated in the vicinity of the metal surface [1]. We have recently reported the deposition of silver nanoparticles into the voids of MOFs using a high-pressure chemical deposition. Via careful choice of the deposition parameters, the particles can be deposited in a range of capillary sizes with their growth being controlled from tens to hundreds of nanometres to tailor the plasmonic properties of the substrate. The resulting metal-dielectric MOFs offer significant benefits over conventional planar detection geometries with the long interaction lengths of the guided modes exciting multiple plasmonic resonances along the fibre.

All measurements were performed using a reflection detection geometry so that the Raman scattered light is collected with same objective lens used to launch the excitation beam. Our initial MOF SERS substrates were fabricated using a $168 \times 12 \mu \mathrm{m}$ hole structure with a $15 \mu \mathrm{m}$ core, as shown in the inset of Fig. 1(a), and were tested for SERS activity using benzenethiol in ethanol (1mM solution followed by a pure ethanol wash). Comparing the SERS spectra obtained by focusing the excitation beam onto the core and then the air hole cladding, there is little difference between the strength of the signals for this structure implying that, due to a poor overlap of the core modes with the metal nanoparticles, the benefits of the long interaction lengths of the guiding modes is traded off by the large silver cross sectional area available to the cladding modes.

Motivated by this we have recently investigated a new MOF template based on a $126 \times 1 \mu \mathrm{m}$ hole structure with a small $2 \mu \mathrm{m}$ silica core, as shown in the inset of Fig. 1(b), where we expect a much larger overlap between the guided modes and the silver particles surrounding the core. Using aminothiophenol as our target molecule, the SERS spectra obtained now show a clear difference in the signal strength for the two excitation geometries with the signal from the core $\sim 10$ times stronger than that from the air hole cladding, highlighting the importance of the long interaction lengths. Significantly, owing to the greatly improved overlap between the excitation beam and the metal nanoparticles, the small core substrates exhibit SERS signals $2 \times 10^{3}$ times stronger than the large core structure. We will discuss the importance of MOF template design and modal overlap engineering to maximise the electromagnetic enhancement factor thus improving the efficiency of the fibre sensors. The optimised substrates have already shown potential for use as remote optical fibre sensors where the SERS active region is confined to the tip of a longer empty MOF.
\end{abstract}
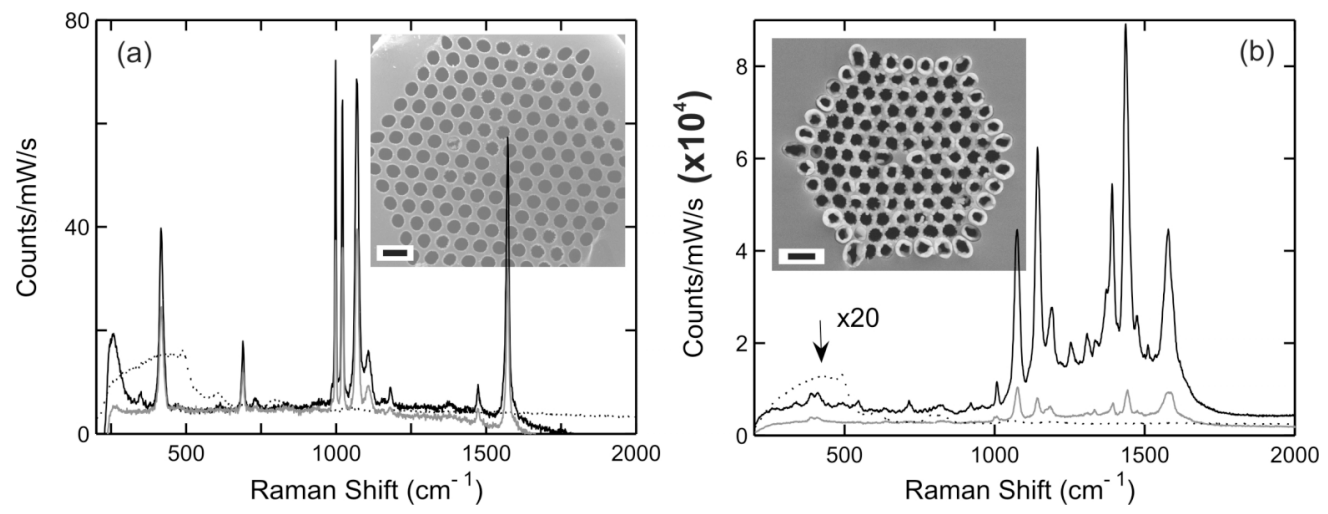

Fig. 1 SERS spectra obtained from the core (black lines) and the cladding (grey lines) of the MOF substrates with (a) large and (b) small silica cores (shown as insets) together with control spectra taken from identical MOFs with no silver filling (dashed lines). Target molecules and scale bars are: (a) benzenethiol, $20 \mu \mathrm{m}$ and (b) aminothiophenol, $2 \mu \mathrm{m}$.

\section{References}

[1] A. Amezcua-Correa et al., Proc. $32^{\text {nd }}$ European Conf. on Optical Comms, Cannes, France, Tu4.3.4 (2006) 